\title{
Retrospective study: clinicopathological features and prognosis of idiopathic membranous nephropathy with seronegative anti-phospholipase $\mathbf{A} 2$ receptor antibody
}

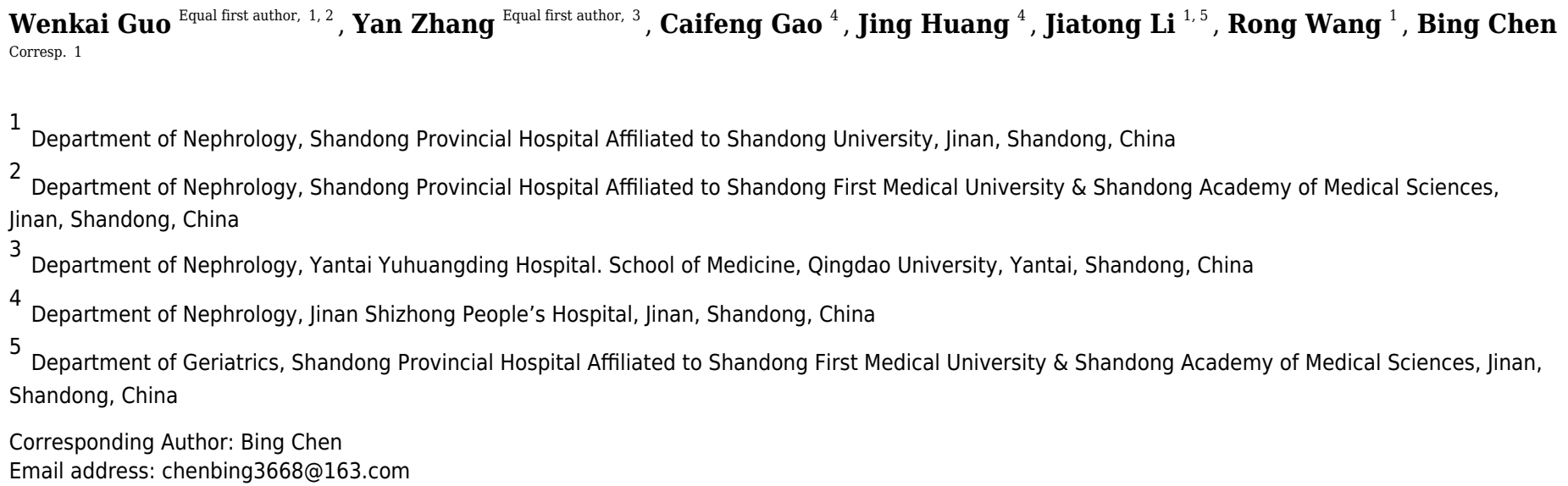

Background: To discuss the clinicopathological features and prognosis of patients with idiopathic membranous nephropathy (IMN) who are serum-negative for the anti-PLA2R antibody.

Method: Overall, 229 IMN patients were retrospectively collected in this study and classified into antiPLA2R antibody-negative (PLA2R-, 59 cases) and antibody-positive (PLA2R+, 170 cases) groups. The clinical and pathological features of the PLA2R-group were analyzed; 162 patients in both groups were followed up, and the PLA2R antigen was detected in renal biopsies from the PLA2R-group. Kaplan-Meier and survival analyses were used to compare differences in prognosis.

Results: Serum albumin levels were higher and 24-hour urine protein, creatinine, and beta 2microglobulin (BMG) levels were lower in the PLA2R-group than in the PLA2R+ group; the proportion of acute and chronic tubular lesions was also significantly lower in the PLA2R-group than in in the PLA2R+ group. After treatment, the remission rate was significantly higher in the negative group than in the positive group $(93.02 \%$ vs $74.78 \%$,), especially the rate of complete remission $(51.16 \%$ vs $23.47 \%$ ). Furthermore, the PLA2R antigen-positive staining rate of 43 patients in the PLA2R-group was $62.79 \%$. Although not significant, the survival rate was higher in the PLA2R-group than in the PLA2R+group. BMG, 24-hour urine protein and acute and chronic tubular lesions were risk factors for kidney death, and 24-hour urine protein was an independent risk factor for kidney death.

Conclusions: Compared with the PLA2R+ group, the PLA2R-group had mild clinical manifestations and pathological damage and a higher clinical treatment remission rate. Renal tissue PLA2R antigen testing can be considered for patients with seronegative IMN to increase the diagnostic rate. 
1 Retrospective study: clinicopathological features and prognosis of idiopathic membranous

2 nephropathy with seronegative anti-phospholipase $\mathrm{A} 2$ receptor antibody

3 Wenkai Guo ${ }^{1,2}+$, Yan Zhang $^{3}{ }^{\dagger}$,Caifeng $\mathrm{Gao}^{4}$, Jing Huang ${ }^{4}$, Jiatong Li ${ }^{1,5}$ Rong Wang ${ }^{1}$

4 ,Bing Chen ${ }^{1 *}$,

5

6 1. Department of Nephrology, Shandong Provincial Hospital Affiliated to Shandong

7 University, Jinan, Shandong, 250001, China

8 2. Department of Nephrology, Shandong Provincial Hospital Affiliated to Shandong First Medical

9 University \& Shandong Academy of Medical Sciences, Jinan, Shandong, 250001, China

10 3. Department of Nephrology, Yantai Yuhuangding Hospital. School of Medicine, Qingdao

11 University, Yantai, Shandong,264000, China

12 4. Department of Nephrology, Jinan Shizhong People's Hospital, Jinan, Shandong, 250001, China

13 5. Department of Geriatrics, Shandong Provincial Hospital Affiliated to Shandong First Medical

14 University \& Shandong Academy of Medical Sciences, Jinan, Shandong, 250001, China

$15 \dagger$ Equal contributors

$16 *$ Corresponding author:

17 Bing Chen

Department of Nephrology, Shandong Provincial Hospital Affiliated to Shandong University, Jinan, Shandong Province, 250001, China

E-mail address: chenbing3668@163.com. 


\section{Abstract}

Background: To discuss the clinicopathological features and prognosis of patients with idiopathic membranous nephropathy (IMN) who are serum-negative for the anti-PLA2R antibody.

Method: Overall, 229 IMN patients were retrospectively collected in this study and classified into anti-PLA2R antibody-negative (PLA2R-, 59 cases) and antibody-positive (PLA2R+, 170 cases) groups. The clinical and pathological features of the PLA2R-group were analyzed; 162 patients in both groups were followed up, and the PLA2R antigen was detected in renal biopsies from the PLA2R-group. Kaplan-Meier and survival analyses were used to compare differences in prognosis.

Results: Serum albumin levels were higher and 24-hour urine protein, creatinine, and beta 2microglobulin (BMG) levels were lower in the PLA2R-group than in the PLA2R+ group; the proportion of acute and chronic tubular lesions was also significantly lower in the PLA2R-group than in in the PLA2R+ group. After treatment, the remission rate was significantly higher in the negative group than in the positive group (93.02\% vs $74.78 \%$,), especially the rate of complete remission (51.16\% vs 23.47\%). Furthermore, the PLA2R antigen-positive staining rate of 43 patients in the PLA2R-group was $62.79 \%$. Although not significant, the survival rate was higher in the PLA2R-group than in the PLA2R+ group. BMG, 24-hour urine protein and acute and chronic tubular lesions were risk factors for kidney death, and 24-hour urine protein was an independent risk factor for kidney death.

Conclusions: Compared with the PLA2R+ group, the PLA2R-group had mild clinical manifestations and pathological damage and a higher clinical treatment remission rate. Renal tissue PLA2R antigen testing can be considered for patients with seronegative IMN to increase the diagnostic rate. 


\section{Introduction}

Membranous nephropathy with unclear etiology is called idiopathic membranous nephropathy (IMN) and accounts for $30 \sim 40 \%$ of all cases of primary nephrotic syndrome (Braden et al. 2000; Couser 2017). Recent clinical observations show that the incidence of membranous nephropathy is increasing, and the age of onset is becoming younger, which may be related to various factors such as improvements in diagnostic factors and environmental pollution (Ayach et al. 2011). Moreover, IMN is also a heterogeneous disease with a long natural course. Approximately one-third of patients will exhibit variable degrees of persistent proteinuria without deterioration in renal function, another third will experience progressive deterioration of renal function and eventually progress to end-stage renal disease (ESRD), and the final third of patients will experience spontaneous remission (Obrisca et al. 2015).

Currently, the method for diagnosing membranous nephropathy is mainly based on renal biopsy, an invasive examination method that has inherent risks and cannot be used to diagnose and treat diseases in a timely and effective manner. Therefore, the serological diagnosis of IMN has become a hot topic in kidney disease research. In 2009, Beck et al. (Beck et al. 2009) discovered autoantibodies against phospholipase A2 receptor antibodies in the glomerular podocytes of IMN patients. PLA2R is a transmembrane protein located on the surface of human podocytes and colocalizes with IgG4 in the immune deposits of glomeruli in patients with IMN. Binding of the circulating anti-PLA2R antibody to the PLA2R antigen on glomerular podocytes to form an in situ immune complex activates a complement to cause podocyte and immune damage that results in urinary protein production, ultimately causing kidney damage (Glassock 2012). Additionally, Beck et al. found that serum anti-PLA2R antibodies were detectable in $70 \%$ of patients with IMN and detected at a low rate in people without kidney disease and other kidney patients (Hofstra \& Wetzels 2014). Further studies have found that the antibody level is related to the urine protein level, disease condition, and prognosis and has high sensitivity and 
specificity in the clinic (Hofstra et al. 2011; Hoxha et al. 2011; Hoxha et al. 2014). In addition to serum PLA2R antibodies, a number of clinical studies (Beck et al. 2009; Dai et al. 2015; Svobodova et al. 2013) have found that the detection of PLA2R antigen in renal tissue also plays an important role in the clinical diagnosis, guiding treatment and judgment of disease activity of IMN. The expression of PLA2R antigen in renal tissue was significantly enhanced in IMN patients, and low or no expression was observed in patients with secondary membranous nephropathy (SMN) and other nonmembranous glomerular diseases, indicating that the PLA2R antigen in renal tissue also has high specificity in the diagnosis of IMN.

Several studies have shown that the anti-PLA2R antibody titer reflects the activity of the clinical disease. The low antibody titer in antibody-positive patients indicates a high disease remission rate, and thus, this antibody can be used as a predictor of clinical efficacy (Hofstra et al. 2012; Oh et al. 2013). However, there are few studies on the clinical and pathological characteristics of patients with IMN who are negative for the serum anti-PLA2R antibody, and the effect of a negative antibody on disease prognosis is unknown. Besides, some studies suggest that patients with negative antibodies may have secondary factors, such as tumors and heavy metals (Radice et al. 2018). Therefore, the characteristics of serum anti-PLA2R antibodynegative IMN require further clinical studies.

In this study, we performed a two-step evaluation. First, we retrospectively compared the clinical and pathological features of anti-PLA2R-negative (PLA2R-) and anti-PLA2R-positive $(\mathrm{PLA} 2 \mathrm{R}+)$ subjects, and the PLA2R antigen was detected in renal biopsies from the PLA2Rgroup. Then, we evaluated the prognostic value of PLA2R-negative results using a longitudinal design and analysis.

\section{Materials and Methods}

\section{Patient selection}


102 From January 2015 to December 2017, 229 adult patients at Shandong Provincial Hospital who were diagnosed with nephrotic syndrome confirmed by renal biopsy as IMN and had complete clinical data were included in this study. All patients had IMN, a condition that is universally diagnosed by kidney biopsy based on the presence of subepithelial spikes along capillary walls as determined by silver staining, granular IgG and C3 along capillary walls as determined by immunofluorescence, and subepithelial deposits as determined by electron microscopy (EM). The clinical study excluded patients with SMN caused by autoimmune disease, tumors, and hepatitis B virus- and metabolic-related diseases. At the time of selection, the patients' renal function was in the normal range, and 162 patients were followed up for more than 6 months with complete follow-up data. This study has obtained verbal consent from patients, as well as reviewed and approved by the Medical Ethics Committee of Shandong Provincial Hospital (LCYJ: no. 2019-105).

\section{Clinical and laboratory data collection}

2.1 General demographics (age, gender) and clinical (duration of disease, systolic and diastolic blood pressure, etc.) records were collected. Laboratory data included general tests (urinary red blood cells, hemoglobin, white blood cells, platelets, alanine aminotransferase (ALT), aspartate aminotransferase (AST), superoxide dismutase (SOD), serum total protein (TP), albumin (ALB), globulin (GLO), cystatin C (Cys-C), retinol-binding protein (RBP), $\beta 2$ microglobulin (BMG), complement C1q, calcium, immunoglobulin, total cholesterol (CHOL), low-density lipoprotein (LDL-C), high-density lipoprotein (HDL-C), antibody, antinuclear antibody, ANCA antibody, blood glucose (GLU), etc.) and specific biomarkers of renal function and disease activity (urine protein, 24-hour urine protein, serum creatinine (CREA), and blood urea nitrogen (BUN)). The eGFR was calculated based on the modification of diet in renal disease (MDRD) formula.

2.2 Anti-PLA2R detection method: The anti-phospholipase A2 receptor antibody IgG detection kit (enzyme-linked immunosorbent assay) produced by EUROIMMUN (product no. EA 1254$9601 \mathrm{G})$ was used and performed according to the standard procedure in the manual using a 
normal reference range of $0-20 \mathrm{RU} / \mathrm{ml}$. The study also included antibody-positive components in the low-titer group (20-180 RU/mL) and high-titer group (> $180 \mathrm{RU} / \mathrm{mL}$ ) (Hofstra et al. 2012).

2.3 PLA2R antigen and IgG4 detection: We collected paraffin sections of kidney biopsies from 43 patients negative for anti-PLA2R antibodies, used direct immunofluorescence to detect the deposition of IgG4 in renal tissue and indirectly detected the PLA2R antigen in renal tissue. The fluorescence intensity was semiquantitatively recorded by fluorescence microscopy and scored as negative (-), suspicious positive $( \pm)$, and positive $(+)$.

\section{Renal biopsy}

Each patient's renal biopsy included at least 10 glomeruli for histopathological evaluation. We performed light microscopy, electron microscopy(EM)and immunofluorescence on renal puncture tissues. Two renal pathologists participated in the reading and review of the pathological results. We divided membranous nephropathy into 4 stages. If two stages were present at the same time, we defined the highest stage as the final stage and stages III and IV as the advanced pathological stages. Simultaneously, this study observed the presence or absence of focal segmental glomerular sclerosis, spheroidal sclerosis, mesangial proliferative lesions, crescent incidence, balloon adhesions, endothelial hyperplasia, acute and chronic tubular lesions, inflammatory cell-infiltrating lesions, small blood vessel lesions, etc. The pathological manifestations of glomerular disease combined with focal segmental glomerulosclerosis (FSGS) showed focal and segmental distribution of glomerular lesions under light microscopy, with an increased mesangial matrix and balloon adhesions as the main manifestations, accompanied by a small amount of mesangial hyperplasia, corresponding tubular atrophy and renal interstitium fibrosis. Spherical sclerosis is defined based on the hardening of one glomerulus, and crescent incidence is defined as the presence of a cellular or fibrous crescent in one glomerulus. Mesangial proliferative disease is defined as the number of cells in the mesangial area being greater than or equal to 4 . Acute tubulointerstitial lesions are defined as tubular epithelial edema or necrosis of at least $10 \%$. Chronic tubulointerstitial lesions are defined as astubular atrophy and 
154

155

156

157

interstitial fibrosis of at least $10 \%$. Interstitial inflammatory disease mainly manifests as neutrophil infiltration. Small vessel lesions include arterial intimal thickening, elastic layer stratification, and hyaline degeneration.

\section{Outcomes in followed up patients}

We defined worsening renal conditions as doubling of the baseline Scr level; we defined ESRD as a creatinine clearance rate of less than $15 \mathrm{ml} / \mathrm{min}$ at the last follow-up, start of dialysis or renal transplantation. Complete remission (CR) was defined as a urine protein level $<0.3 \mathrm{~g} / \mathrm{d}$ based on the premise of stable renal function. Partial remission (PR) was defined as a $50 \%$ reduction in the urine protein to a proteinuria level $<3.5 \mathrm{~g} / \mathrm{d}$. A simplified MDRD formula was used to calculate eGFR as follows: eGFR $\left[\mathrm{ml} \cdot \mathrm{min}^{-1} \cdot\left(1.73 \mathrm{~m}^{2}\right)^{-1}\right]=186 \times[\mathrm{Scr}(\mu \mathrm{mol} / \mathrm{L}) / 88.4]^{-1.154} \times \mathrm{Age}^{-0.203 \times(\text { if }}$ female, 0.742). Serious complications included clinical death, serious lung infection, pulmonary embolism, cerebral infarction, myocardial infarction, and tumors. All patients underwent eGFR measurements at the beginning and after treatment.

\section{Statistical methods}

Statistical analysis was performed using the statistical software SPSS 19.0. Data with a normal distribution were expressed as mean \pm standard deviation (SD) and compared by t-tests, and data with a non-normal distribution were presented as the median and quartile and compared by nonparametric test. The categorical variables were expressed as rates and were compared by the $\chi 2$ test. Correlation between several parameters was expressed as Spearman rank coefficient of correlation. Patient and renal survival probabilities were estimated by the Kaplan- Meier method. The relationships of the covariates to patient and renal survival were evaluated in the univariate analysis with the log-rank test and in the multivariate analysis with the Cox proportional hazards model; the Harrel C statistic was used for verification of the risk factors confirmed by the Cox proportional hazards model. A Cox analysis of risk factors was performed in combination with clinical significance. $\mathrm{P}<0.05$ was considered statistically significant. 


\section{Results}

1. General clinical data: Two hundred twenty-nine patients with IMN were retrospectively collected and analyzed in this study, including 59 (25.76\%) patients in the PLA2R-group and $170(74.24 \%)$ patients in the PLA2R + group. Among the antibody-positive group, there were $109(64.12 \%)$ patients in the low-titer group and $61(35.88 \%)$ patients in the high-titer group. The comparisons of baseline clinical characteristics and laboratory examination data between the two groups are shown in Table 1. In this study, the levels of serum creatinine $(61.57 \pm 15.88 \mathrm{vs}$ $65.90 \pm 15.64, \mathrm{df}=226, \mathrm{P}=0.033)$ and $\beta 2$-microglobulin $(2.13 \pm 0.74$ vs $2.39 \pm 0.81, \mathrm{df}=209$, $\mathrm{P}=0.045)$ in the PLA2R-group were lower than those in the PLA2R+ group, and the level of ALB $(26.11 \pm 6.02$ vs $24.20 \pm 5.11, \mathrm{df}=226, \mathrm{P}=0.019)$ in the PLA2R-group was higher than that in the PLA2R+ group. The average 24-hour urine protein quantification in the PLA2R-group was slightly lower than that in the PLA2R+ group (4.91 \pm 3.18 vs $5.81 \pm 3.50)$, but the difference was not statistically significant $(\mathrm{df}=224, \mathrm{P}=0.086)$. At the same time, we found no correlation between the anti-PLA2R antibody level and the serum creatinine level in the PLA2R+ patients $(\mathrm{r}=-0.08, \mathrm{P}=0.256)$. The anti-PLA2R antibody level was positively correlated with proteinuria $(\mathrm{r}=0.151, \mathrm{P}=0.05)$ and negatively correlated with serum albumin $(r=-0.164, \mathrm{P}=0.03)$. The 24hour urine protein levels of patients in the low-titer group were significantly lower than those in patients in the high-titer group $(5.28 \pm 3.42$ vs $6.76 \pm 3.46, \mathrm{df}=166, \mathrm{P}=0.008)$. The eGFR values of the antibody-negative and antibody-positive groups were 115.394 and $109.860[\mathrm{ml} \cdot \mathrm{min}-1 \cdot(1.73$ $\mathrm{m} 2)-1]$, respectively, but the difference was not statistically significant $(\mathrm{df}=225, \mathrm{P}=0.172$ ).

2. Pathological data: Renal biopsy immunofluorescence showed no significant differences in IgG, IgM, IgA, C3, Fib, or C1q deposits between the two groups. There was no significant difference in the proportion of renal pathological stages between the two groups $(\mathrm{P}>0.05)$. The proportions of acute and chronic tubular lesions and inflammatory cell infiltration in the PLA2Rgroup were significantly lower than those in the positive group (respectively, $3.39 \%$ vs $11.76 \%$ 
204

205

206

207

208

209

210

211

212

213

214

215

216

217

218

219

220

221

222

223

224

225

226

227

228

229

230

$\mathrm{df}=180, \mathrm{P}=0.015 ; 3.39 \%$ vs $13.53 \% \mathrm{df}=183, \mathrm{P}=0.01$; and $5.08 \%$ vs $13.53 \% \mathrm{df}=158, \mathrm{P}=0.031$ )

(Table 2).

3. Treatment and outcomes: Among the 229 patients, 162 patients, including 43 patients in the PLA2R-group and 119 patients in the PLA2R+ group, were followed up for more than 6 months, and the average follow-up time was $11.97 \pm 3.84$ months. We treated patients with individualized and specific treatments, including glucocorticoids plus immunosuppressants (cyclophosphamide (CTX), cyclosporine A (CSA), tacrolimus). Our treatment plans were as follows: patients on drug regimens were treated according to their blood pressure and blood lipids. Acetylcholinesterase inhibitors (ACEIs) and angiotensin type 1 receptor blockers (ARBs) were used to control blood pressure below 140/90 $\mathrm{mmHg}$, and statins were used to control blood lipids. Depending on the presence of edema, diuretics were sometimes used. Tacrolimus was administered according to the following regimen: an initial oral dose of $0.5 \mathrm{mg} /(\mathrm{kg} \cdot \mathrm{d})$, with continued treatment for at least 6 months. The plasma concentration of tacrolimus was determined for 1 week to maintain of value of $5 \sim 10 \mathrm{ng} / \mathrm{ml}$. CSA was administered according to the following regimen: an initial oral dose of $3-5 \mathrm{mg} /(\mathrm{kg} \cdot \mathrm{d})$, with continued treatment for at least 6 months. The plasma concentration of CsA was monitored for 1 week to maintain a value of 100 200 ng/ml. CTX was administered according to the following regimen: administration of a static dose of $750 \mathrm{mg} / \mathrm{m} 2$ body surface area for at least 6 months and a cumulative dose of 6-8 g. All patients were given a sufficient dose of prednisone $1 \mathrm{mg} /(\mathrm{kg} \cdot \mathrm{d})$. After 8 weeks of adequate treatment, the dose size was reduced by $5 \mathrm{mg}$ every 2 weeks and then held constant at a low dose of $10 \mathrm{mg} / \mathrm{d}$. The total course of treatment was at least 9 months. This study found that the overall remission rate (including CR and PR) in the PLA2R-group was 93.02\%, which was significantly higher than that in the PLA2R+ group (74.78\%), and the difference was statistically significant $(\chi 2=6.474, \mathrm{df}=1, \mathrm{P}=0.01)$. The $\mathrm{CR}$ and $\mathrm{PR}$ rates in the negative group were $51.16 \%$ and $41.86 \%$, respectively, and the $\mathrm{CR}$ and $\mathrm{PR}$ rates in the positive group were $24.37 \%$ and $50.42 \%$. In addition, in the antibody-positive group, the low titer group had a higher remission rate than the antibody high titer group(81.58\%VS62.79\%), and the difference was statistically significant 
$231(\chi 2=5.142, \mathrm{df}=1, \mathrm{P}=0.02)$. We further performed a subgroup analysis, which revealed that the 232 CR rate of patients in the PLA2R-group was significantly higher than that of patients in the 233 PLA2R + group in the different treatment regimens (Table 3). Consistent with the above results, 234 the serum albumin levels of both groups were increased, and the quantified 24-hour urine protein 235 levels were decreased significantly after treatment. However, the levels of proteinuria $(\mathrm{df}=154$, 236 $\mathrm{P}=0.01)$, serum creatinine and urea nitrogen $(\mathrm{df}=106, \mathrm{P}=0.03)$ in the negative group were lower than those in the positive group, and the albumin level $(\mathrm{df}=156, \mathrm{P}=0.036)$ in the negative group was higher than that in the positive group (Table 4). After treatment, the eGFR values of the negative and positive groups were $107.77 \pm 24.431$ and $105.20 \pm 28.09$ [ml $\cdot \mathrm{min}-1 \cdot(1.73 \mathrm{~m} 2)-1]$, respectively, but the difference was not statistically significant. Moreover, for patients with IMN with follow-up data, the reduction rate of the anti-pla2r antibody after treatment was $92 \%$. In addition, we detected both the PLA2R antigen and IgG4 in the renal tissues of 43 patients with negative antibodies by staining, revealing that 27 (62.79\%) people were positive for the PLA2R antigen, 29 (67.44\%) people were positive for IgG4, and 25 (58.14\%) people were positive for both (Figure 1). By the end of the follow-up, a total of 5 patients had worsening renal conditions, and all 5 patients were in the PLA2R+ group. Four of the patients were in the hormone plus calcineurin inhibitor treatment group, and of these, 2 patients had excessively high blood drug concentrations, 2 patients had some relief of proteinuria, and 2 patients had no improvement of proteinuria. Another patient was assigned to the hormone plus CTX group, and the patient's proteinuria did not turn negative. During the follow-up process, we found one case of a benign lung tumor and three cases of severe complications, including 2 cases of severe pneumonia (1 death without renal failure) and 1 case of intracranial fungal infection, in the PLA2R+group (Table 5).

In this study, we used the Kaplan-Meier method to map the survival curve. The log-rank test showed no significant difference in renal survival between the two groups $(\chi 2=1.586, \mathrm{P}=0.208)$ (Figure 2). At the same time, we conducted risk factor analysis, revealing that BMG, 24-hour 257 urine protein and acute and chronic tubular lesions were risk factors for kidney death. The Cox 
258 proportional hazard model analysis showed that 24-hour urine protein was an independent risk 259 factor for renal death $(\mathrm{P}=0.034)$ (Tables 6, 7).

260

261

262

263

264

265

266

267

268

269

270

271

272

273

274

275

276

277

278

279

280

281

282

283

\section{Discussion}

IMN is an autoimmune disease caused by endogenous antigens combined with specific antibodies in the circulation to form in situ immune complexes that are deposited in renal tissues. The antiPLA2R antibody in circulation binds to PLA2R on the surface of podocytes to form an electrondense deposit under the foot process and epithelium, thereby activating the complement-forming membrane attack complex, causing podocyte and kidney damage (Glassock 2012). Several studies have demonstrated that the anti-PLA2R antibody is a key factor in the pathogenesis of IMN. However, there are few studies on patients with IMN who are negative for the serum anti-PLA2R antibody, and the pathogenesis and prognosis of patients with IMN who are negative for the antiPLA2R antibody are not very clear. In this study, we first studied the clinicopathological features and prognosis of IMN patients who were seronegative for the anti-PLA2R antibody.

Herein, $74.23 \%$ of the 229 patients with IMN were positive for the antibody, which was consistent with previous studies, as the rate generally ranges from $71.0-77.8 \%$ in IMN patients (Kanigicherla et al. 2013). The 24-hour urine protein, BMG, and creatinine levels were significantly lower in the negative group than in the positive group, and plasma albumin levels were higher in the negative group than in the positive group. Other clinical studies also showed that patients in the PLA2R-group had lower 24-hour urine protein and higher albumin levels than those in the antibody-positive group, which is basically consistent with our findings (Hofstra et al. 2011; Oh et al. 2013; Radice et al. 2016). In this study, the antibody titer in the positive group was positively correlated with the 24-hour urine protein level and negatively correlated with the serum albumin level. J. M. Hofstra et al (Hofstra et al. 2011) also showed that the anti-PLA2R antibody level was positively correlated with the urine protein level and negatively correlated with the albumin level. However, their study also showed a positive correlation between antibodies and serum creatinine, which was inconsistent with the results of our study. The reason for this 
284

285

286

287

288

289

290

291

292

293

294

295

296

297

298

299

300

301

302

303

304

305

306

307

308

309

310

discrepancy may be related to the facts that the renal function of the patients in our study was within the normal range and that the clinical symptoms were relatively mild. In terms of renal pathology, the incidence of acute and chronic tubular injury was significantly lower in the negative group than in the positive group, which was consistent with the results of Hara et al. (Hihara et al. 2016) and indicates that the pathological damage of patients in the antibody-negative group is less severe than that of patients in the positive group.

In our study, we selected patients with an average daily urine protein level $\geq 6 \mathrm{~g}$, and these patients had at least a moderate or high risk of progressive disease. In addition, CR can lead to a good long-term prognosis, and PR can independently reduce the risk of renal failure (Troyanov et al. 2004). Therefore, early therapeutic intervention may be a more effective approach for treating IMN patients with severe proteinuria. For newly diagnosed patients, on the basis of adjuvant therapies such as antihypertensive, lipid-lowering and anticoagulative treatments, we preferred hormone therapy with CTX or a semiquantitative hormone plus tacrolimus (FK506) and CSA. Herein, the remission rate of the negative group was significantly higher than that of the positive group, especially the CR rate. To the best of our knowledge, this study is the first to report that patients in the negative group had a better clinical remission rate than those in the positive group. Furthermore, in the positive group, patients with a low titer had low 24-hour proteinuria levels, high albumin levels, mild pathological damage, and higher remission rates than those with a high antibody titer, thus suggesting that the patients with low antibody titers were more likely to achieve clinical remission. Lida M Rodas et al. (Rodas et al. 2019) found that spontaneous CR did not occur in patients with PLA2R levels $>40 \mathrm{IU} / \mathrm{mL}$ and was less frequently observed in patients with a proteinuria level $>8 \mathrm{~g} /$ day. Furthermore, patients with high antibody levels had a higher risk of developing ESRD. The authors also found that patients with low anti-PLA2R antibody titers (especially antibody titers $<40 \mathrm{UI} / \mathrm{mL}$ ) and proteinuria levels $<4 \mathrm{~g}$ /day had high spontaneous remission rates. These studies are consistent with our findings, indicating that the anti-PLA2R antibodies titer is strongly correlated with the disease activity of IMN.

Survival analysis in this study showed that the survival rate of the PLA2R-group was higher 
311 than that of the PLA2R+ group, but this difference was not statistically significant. Risk factor 312 analysis results showed that BMG, 24-hour urine protein, acute and chronic tubular disease were 313 risk factors, but only 24-hour urine protein was an independent risk factor for renal death. In a 314 predictive model of IMN progression risk, Cattran et al. (Cattran et al. 1997; Pei et al. 1992) 315 proposed that patients with abnormal or worsening serum creatinine or proteinuria, especially 316 those with a duration exceeding 6 months, had a 72\% chance of developing end-stage renal failure.

317 Shiiki H and Ponticelli C et al. (Ponticelli et al. 1989; Shiiki et al. 2004) also showed that high 318 serum creatinine concentrations, severe proteinuria, and chronic tubulointerstitial lesions were important predictors of the risk of IMN progressing to ESRD. Various clinical indicators, such as severe proteinuria, decreased renal function at diagnosis, and development of renal tubular interstitial lesions, can be considered risk factors for the progression of IMN to renal failure. Herein, 43 of 162 IMN patients were negative for the serum anti-PLA2R antibody, potentially due to the following reasons: 1 . The serum anti-PLA2R antibody was negative, but the PLA2R antigen in kidney tissue was positive; 2. other pathogenic antigens and specific autoantibodies may have existed in the patients with IMN; 3. other secondary factors that have not yet been discovered played roles; 4. the anti-PLA2R antibody in the sera of patients with IMN was indeed negative; 5. the patients' conditions were in a stable state, and there was no immune activity in the body; and 6. the limitations of the detection technology itself. Therefore, we stained for the PLA2R antigen in the kidney tissues of 43 patients who were negative for the serum anti-PLA2R antibody, and the granular staining rate for the PLA2R antigen in kidney tissue was $62.79 \%$. Consequently, for patients with IMN who were negative for serum anti-PLA2R antibodies, we could routinely perform renal tissue PLA2R antigen staining. This result is also consistent with the findings of Hill et al. (Hill et al. 2016), who found that the PLA2R antigen was potentially positive in renal tissue when serum antibodies were absent in IMN patients, and when the serum anti-PLA2R antibody was detected in combination with the PLA2R antigen in renal tissue, the sensitivity of disease diagnosis could be increased to $95.2 \%$. Detection of the PLA2R antigen in renal tissue is more sensitive and specific for the diagnosis of IMN and can be used as a supplementary detection 
338

339

340

341

342

343

344

345

346

347

348

349

350

351

352

353

354

355

356

357

358

359

360

361

362

363

364

method for patients who have undergone renal biopsy and are negative for the serum anti-PLA2R antibody (Hofstra \& Wetzels 2014; Svobodova et al. 2013).

For patients with IMN who were negative for the anti-PLA2R antibody, we also considered the presence of other antibodies with structural functions similar to those of the anti-PLA2R antibody. Several clinical reports on other pathogenic antigens of IMN, including thrombospondin type-1 domain-containing 7A (THSD7A) (Tomas et al. 2014), superoxide dismutase (SOD2), $\alpha$-enolase (a-ENO), aldose reductase (AR) (Bruschi et al. 2011; Prunotto et al. 2010), neutral endopeptidase (NEP), bovine serum albumin (BSA) and BSA antibodies (Murtas et al. 2012), have been published. In 2014, Tomas et al. found THSD7A in the sera of patients with IMN who were negative for the PLA2R antibody. This study suggested that antibodies specific for this antigen were detectable only in the sera of anti-PLA2R antibody-negative IMN patients and were almost undetectable in patients with SMN and other glomerular diseases. The anti-THSD7A antibody may become a highly specific new indicator of disease diagnosis and activity monitoring in IMN patients who are negative for the serum anti-PLA2R antibody (Tomas et al. 2014). In addition, some studies showed that the serum anti-PLA2R antibody, anti-THSD7A antibody, anti-SOD2 antibody, and anti-ENO antibody were all negative in IMN patients with a low proportion of nephrotic syndrome at onset, high plasma albumin level, and high disease remission rate (Murtas et al. 2012). Therefore, the anti-THSD7A antibody, anti-SOD2 antibody, and anti- $\alpha$ ENO antibody in the sera of patients with IMN who are negative for the anti-PLA2R antibody can potentially indicate that the patient's clinical severity is less severe, and clinical remission can be achieved more quickly. We also need to conduct a larger number of clinical data studies in the future to confirm these results. In this study, we did not find new tumors in the antibody-negative group, and a benign lung tumor occurred in one patient in the antibody-positive group. This finding was not exactly the same as the relevant research results. Previous clinical studies showed that patients with IMN were negative for the anti-PLA2R antibody, especially when they had antibodies against THSD7A. Furthermore, 21\% of patients with IMN associated with THSD7A were found to have tumors within 3 months of being diagnosed with membranous nephropathy (Hoxha et al. 2016). 
Qin W et al. detected serum anti-PLA2R autoantibodies in 10 patients with tumor-associated membranous nephropathy. Seven of the 10 patients were negative for the anti-PLA2R antibody, while only 3 patients were positive, and most patients with tumor-associated membranous nephropathy were considered to be negative for the antibody in the study (Qin et al. 2011). In the antibody-negative group in our study, there were no tumors (low tumor incidence rate), the clinical remission rate was high, and the prognosis was good. The reasons for our results may be related to the short follow-up time, loss of follow-up, and other pathogenic factors. We also need to further follow-up with the negative group to observe the relationship between tumors and the anti-PLA2R antibody.

Immunosuppressive therapy has been well proven in patients with IMN. However, because this treatment regimen may cause potential side effects, it must be reserved for patients who truly benefit from this treatment. This retrospective study also found that patients with IMN who were negative for the anti-PLA2R antibody had fewer clinical manifestations and renal pathological changes, and numerous side effects of early immunosuppressive therapy were observed in these patients. Therefore, whether patients with IMN who are negative for the anti-PLA2R antibody and without tumors and other secondary factors can actively use immunosuppressive therapy at an early stage to achieve disease remission will be a new direction of our future research. In addition, for patients with IMN who are negative for the anti-PLA2R antibody, if other specific antibodies are not detected in their serum and the renal tissue PLA2R antigen staining result is also negative, we should consider whether other secondary findings exist that have not yet been discovered, such as tumors, heavy metals, and metabolic factors.

\section{Conclusions}

This study focused on the clinical significance of negativity for the serum anti-PLA2R antibody in IMN. The patients who were negative for the serum anti-PLA2R antibody had mild clinical manifestations, mild pathological damage, and relatively high clinical remission rates. Renal tissue PLA2R antigen testing can be considered for patients with seronegative IMN to increase the 
diagnostic rate of IMN. In addition, studies have shown that 24-hour urine protein is an independent risk factor for kidney death. This retrospective study has some limitations, as the study sample size was limited, and the follow-up time was relatively short. The long-term prognosis and factors related to survival analysis need to be further studied.

\section{ACKNOWLEDGMENTS}

We thank Yongmei Wang, Shiyin Jiang, Ruihua Song and Shimin Zhao for their help with data collection.

\section{Ethics approval and consent to participate}

The collection of data from related cases was approved by the Ethics

Committee of Shandong Provincial Hospital Affiliated to Shandong

University (LCYJ: no. 2019-105).

\section{References}

Ayach G, El-Filali H, Saidi S, El-Gharib S, Kaobai G, Noaur H, El-Khayat S, Zamed M, Medkouri G, Hachim K, Benghanem M, and Ramdani B. 2011. Histopathological study of pure primary nephrotic syndrome in adolescents and young Moroccan adults. Arab J Nephrol Transplant 4:137-140.

Beck LH, Bonegio RG, Lambeau G, Beck DM, Powell DW, Cummins TD, Klein JB, and Salant DJ. 2009. M-type phospholipase A2 receptor as target antigen in idiopathic membranous nephropathy. $N$ Engl J Med 361:1121. 10.1056/NEJMoa0810457

Braden GL, Mulhern JG, O'Shea MH, Nash SV, Ucci AA, and Germain MJ. 2000. Changing incidence of glomerular diseases in adults. Am J Kidney Dis 35:878-883.

Bruschi M, Carnevali ML, Murtas C, Candiano G, Petretto A, Prunotto M, Gatti R, Argentiero L, Magistroni R, Garibotto G, Scolari F, Ravani P, Gesualdo L, Allegri L, and Ghiggeri GM. 2011. Direct characterization of target podocyte antigens and auto-antibodies in human membranous glomerulonephritis: Alfa-enolase and borderline antigens. J Proteomics 74:2008-2017. 10.1016/j.jprot.2011.05.021

Cattran DC, Pei Y, Greenwood CM, Ponticelli C, Passerini P, and Honkanen E. 1997. Validation of a predictive model of idiopathic membranous nephropathy: its clinical and research implications. Kidney Int 51:901907.

Couser WG. 2017. Primary Membranous Nephropathy. Clin J Am Soc Nephrol 12:983-997. 10.2215/CJN.11761116

Dai H, Zhang H, and He Y. 2015. Diagnostic accuracy of PLA2R autoantibodies and glomerular staining for the differentiation of idiopathic and secondary membranous nephropathy: an updated meta-analysis. Sci Rep 
5:8803. $10.1038 /$ srep08803

Glassock RJ. 2012. The pathogenesis of membranous nephropathy: evolution and revolution. Curr Opin Nephrol Hypertens 21:235-242. 10.1097/MNH.0b013e3283522ea8

Hihara K, Iyoda M, Tachibana S, Iseri K, Saito T, Yamamoto Y, Suzuki T, Wada Y, Matsumoto K, and Shibata T. 2016. Anti-Phospholipase A2 Receptor (PLA2R) Antibody and Glomerular PLA2R Expression in Japanese Patients with Membranous Nephropathy. PLoS One 11:e0158154. 10.1371/journal.pone.0158154

Hill PA, McRae JL, and Dwyer KM. 2016. PLA2R and membranous nephropathy: A 3 year prospective Australian study. Nephrology (Carlton) 21:397-403. 10.1111/nep.12624

Hofstra JM, Beck LH, Beck DM, Wetzels JF, and Salant DJ. 2011. Anti-phospholipase $\mathrm{A}_{2}$ receptor antibodies correlate with clinical status in idiopathic membranous nephropathy. Clin J Am Soc Nephrol 6:1286-1291. 10.2215/CJN.07210810

Hofstra JM, Debiec H, Short CD, Pellé T, Kleta R, Mathieson PW, Ronco P, Brenchley PE, and Wetzels JF. 2012. Antiphospholipase $\mathrm{A} 2$ receptor antibody titer and subclass in idiopathic membranous nephropathy. $J \mathrm{Am}$ Soc Nephrol 23:1735-1743. 10.1681/ASN.2012030242

Hofstra JM, and Wetzels JF. 2014. Phospholipase A2 receptor antibodies in membranous nephropathy: unresolved issues. J Am Soc Nephrol 25:1137-1139. 10.1681/ASN.2014010091

Hoxha E, Harendza S, Zahner G, Panzer U, Steinmetz O, Fechner K, Helmchen U, and Stahl RA. 2011. An immunofluorescence test for phospholipase- $\mathrm{A}_{2}$-receptor antibodies and its clinical usefulness in patients with membranous glomerulonephritis. Nephrol Dial Transplant 26:2526-2532. 10.1093/ndt/gfr247

Hoxha E, Thiele I, Zahner G, Panzer U, Harendza S, and Stahl RA. 2014. Phospholipase A2 receptor autoantibodies and clinical outcome in patients with primary membranous nephropathy. J Am Soc Nephrol 25:1357-1366. 10.1681/ASN.2013040430

Hoxha E, Wiech T, Stahl PR, Zahner G, Tomas NM, Meyer-Schwesinger C, Wenzel U, Janneck M, Steinmetz OM, Panzer U, Harendza S, and Stahl RA. 2016. A Mechanism for Cancer-Associated Membranous Nephropathy. N Engl J Med 374:1995-1996. 10.1056/NEJMc1511702

Kanigicherla D, Gummadova J, McKenzie EA, Roberts SA, Harris S, Nikam M, Poulton K, McWilliam L, Short CD, Venning M, and Brenchley PE. 2013. Anti-PLA2R antibodies measured by ELISA predict long-term outcome in a prevalent population of patients with idiopathic membranous nephropathy. Kidney Int 83:940948. 10.1038/ki.2012.486

Murtas C, Bruschi M, Candiano G, Moroni G, Magistroni R, Magnano A, Bruno F, Radice A, Furci L, Argentiero L, Carnevali ML, Messa P, Scolari F, Sinico RA, Gesualdo L, Fervenza FC, Allegri L, Ravani P, and Ghiggeri GM. 2012. Coexistence of different circulating anti-podocyte antibodies in membranous nephropathy. Clin J Am Soc Nephrol 7:1394-1400. 10.2215/CJN.02170312

Obrisca B, Ismail G, Jurubita R, Baston C, Andronesi A, and Mircescu G. 2015. Antiphospholipase A2 Receptor Autoantibodies: A Step Forward in the Management of Primary Membranous Nephropathy. Biomed Res Int 2015:249740. 10.1155/2015/249740

Oh YJ, Yang SH, Kim DK, Kang SW, and Kim YS. 2013. Autoantibodies against phospholipase A2 receptor in Korean patients with membranous nephropathy. PLoS One 8:e62151. 10.1371/journal.pone.0062151

Pei Y, Cattran D, and Greenwood C. 1992. Predicting chronic renal insufficiency in idiopathic membranous glomerulonephritis. Kidney Int 42:960-966.

Ponticelli C, Zucchelli P, Passerini P, Cagnoli L, Cesana B, Pozzi C, Pasquali S, Imbasciati E, Grassi C, and 
466

467

468

469

470

471

472

473

474

475

476

477

478

479

480

481

482

483

484

485

486

487

488

489

490

491

492

493

494

495

496

497

498

499

500

501

Redaelli B. 1989. A randomized trial of methylprednisolone and chlorambucil in idiopathic membranous nephropathy. N Engl J Med 320:8-13. 10.1056/NEJM198901053200102

Prunotto M, Carnevali ML, Candiano G, Murtas C, Bruschi M, Corradini E, Trivelli A, Magnasco A, Petretto A, Santucci L, Mattei S, Gatti R, Scolari F, Kador P, Allegri L, and Ghiggeri GM. 2010. Autoimmunity in membranous nephropathy targets aldose reductase and SOD2. J Am Soc Nephrol 21:507-519. 10.1681/ASN.2008121259

Qin W, Beck LH, Zeng C, Chen Z, Li S, Zuo K, Salant DJ, and Liu Z. 2011. Anti-phospholipase A2 receptor antibody in membranous nephropathy. J Am Soc Nephrol 22:1137-1143. 10.1681/ASN.2010090967

Radice A, Pieruzzi F, Trezzi B, Ghiggeri G, Napodano P, D'Amico M, Stellato T, Brugnano R, Ravera F, Rolla D, Pesce G, Giovenzana ME, Londrino F, Cantaluppi V, Pregnolato F, Volpi A, Rombolà G, Moroni G, Ortisi G, and Sinico RA. 2018. Diagnostic specificity of autoantibodies to M-type phospholipase A2 receptor (PLA2R) in differentiating idiopathic membranous nephropathy (IMN) from secondary forms and other glomerular diseases. J Nephrol 31:271-278. 10.1007/s40620-017-0451-5

Radice A, Trezzi B, Maggiore U, Pregnolato F, Stellato T, Napodano P, Rolla D, Pesce G, D'Amico M, Santoro D, Londrino F, Ravera F, Ortisi G, and Sinico RA. 2016. Clinical usefulness of autoantibodies to M-type phospholipase A2 receptor (PLA2R) for monitoring disease activity in idiopathic membranous nephropathy (IMN). Autoimmun Rev 15:146-154. 10.1016/j.autrev.2015.10.004

Rodas LM, Matas-García A, Barros X, Blasco M, Viñas O, Llobell A, Martin N, and Quintana LF. 2019. Antiphospholipase 2 receptor antibody levels to predict complete spontaneous remission in primary membranous nephropathy. Clin Kidney J 12:36-41. 10.1093/ckj/sfy005

Shiiki H, Saito T, Nishitani Y, Mitarai T, Yorioka N, Yoshimura A, Yokoyama H, Nishi S, Tomino Y, Kurokawa K, Sakai H, and Japan RGoPRDi. 2004. Prognosis and risk factors for idiopathic membranous nephropathy with nephrotic syndrome in Japan. Kidney Int 65:1400-1407. 10.1111/j.1523-1755.2004.00518.x

Svobodova B, Honsova E, Ronco P, Tesar V, and Debiec H. 2013. Kidney biopsy is a sensitive tool for retrospective diagnosis of PLA2R-related membranous nephropathy. Nephrol Dial Transplant 28:1839-1844. $10.1093 / \mathrm{ndt} / \mathrm{gfs} 439$

Tomas NM, Beck LH, Meyer-Schwesinger C, Seitz-Polski B, Ma H, Zahner G, Dolla G, Hoxha E, Helmchen U, Dabert-Gay AS, Debayle D, Merchant M, Klein J, Salant DJ, Stahl RAK, and Lambeau G. 2014. Thrombospondin type-1 domain-containing 7A in idiopathic membranous nephropathy. $N$ Engl J Med 371:2277-2287. 10.1056/NEJMoa1409354

Troyanov S, Wall CA, Miller JA, Scholey JW, Cattran DC, and Group TGR. 2004. Idiopathic membranous nephropathy: definition and relevance of a partial remission. Kidney Int 66:1199-1205. 10.1111/j.15231755.2004.00873.x

Peer] reviewing PDF | (2019:08:40799:3:0:NEW 17 Jan 2020) 


\section{Table $\mathbf{1}$ (on next page)}

Comparison of clinical characteristics and basic laboratory tests between the two groups

Note: SBP, systolic blood pressure; DBP, diastolic blood pressure; 24HUPRO, 24-hour urine;

WBC, white blood cells; PLT, platelets; HGB, hemoglobin; AST, alanine aminotransferase; AST, aspartate aminotransferase; SOD, superoxide dismutase; TP, serum total protein; ALB, albumin; GLO, globulin; GLU, blood glucose; BUN, blood urea nitrogen; CREA, serum creatinine; Cys-C, cystatin C; BMG, $\beta 2$ macroglobulin; RBP, retinol binding protein; $\mathrm{CHOL}$, total cholesterol; HDL-C, high-density lipoprotein; LDL-C, low-density lipoprotein; FSGS, focal glomerular sclerosis; IgG4, immunoglobulin G4; IgG, immunoglobulin G; IgM, immunoglobulin M; IgA, immunoglobulin A; C3 C4, complement 3 complement 4;PLA2R, phospholipase A2 receptors. Compared with the PLa2R- group and the PLA2R+group, $\mathrm{P} *<0.05, \mathrm{P} * *<0.01$. 
1 Table 1.

\begin{tabular}{|c|c|c|c|c|}
\hline & $\operatorname{IMN}(\mathrm{N}=229)$ & $\begin{array}{l}\text { APLA2R-ab(- } \\
)(\mathrm{N}=59)\end{array}$ & APLA2R-ab $+(N=170)$ & P value \\
\hline Age (year) & $45.88 \pm 13.64$ & $45.75 \pm 13.49$ & $45.92 \pm 13.73$ & 0.931 \\
\hline Sex (female $(\%)$ ) & $91 / 229(39.74 \%)$ & $30 / 59(50.84 \%)$ & $61 / 170(35.88 \%)$ & $0.043^{*}$ \\
\hline $\begin{array}{l}\text { Duration of illness } \\
\text { (month) }\end{array}$ & $117.72 \pm 175.40$ & $129.57 \pm 210.58$ & $112.44 \pm 160.84$ & 0.542 \\
\hline $\mathrm{SBP}(\mathrm{mmHg})$ & $137.70 \pm 20.48$ & $135.63 \pm 23.30$ & $140.90 \pm 21.03$ & 0.190 \\
\hline DBP (mmHg) & $87.10 \pm 13.89$ & $88.93 \pm 16.66$ & $88.08 \pm 13.93$ & 0.626 \\
\hline Urine $\mathrm{RBC} / \mathrm{HPF}$ & $14.07 \pm 17.76$ & $11.24 \pm 14.87$ & $15.04 \pm 18.59$ & 0.160 \\
\hline 24HUPRO (g/L) & $6.12 \pm 8.17$ & $4.91 \pm 3.18$ & $5.81 \pm 3.50$ & 0.086 \\
\hline $\mathrm{WBC}\left(\times 10^{\wedge} 9 / \mathrm{L}\right)$ & $8.28 \pm 17.76$ & $11.95 \pm 34.47$ & $7.00 \pm 2.81$ & 0.275 \\
\hline HGB (g/L) & $134.28 \pm 30.47$ & $132.18 \pm 38.80$ & $135.01 \pm 27.05$ & 0.605 \\
\hline $\operatorname{PLT}\left(\times 10^{\wedge} 9 / \mathrm{L}\right)$ & $268.09 \pm 71.73$ & $270.17 \pm 95.40$ & $267.36 \pm 61.64$ & 0.833 \\
\hline $\operatorname{AST}(\mathrm{u} / \mathrm{L})$ & $22.67 \pm 8.20$ & $23.26 \pm 8.61$ & $22.46 \pm 8.07$ & 0.525 \\
\hline $\operatorname{ALT}(\mathrm{u} / \mathrm{L})$ & $22.83 \pm 11.96$ & $25.37 \pm 12.18$ & $21.96 \pm 11.79$ & 0.063 \\
\hline $\operatorname{SOD}(\mathrm{u} / \mathrm{mL})$ & $111.83 \pm 27.18$ & $115.83 \pm 28.70$ & $110.41 \pm 26.59$ & 0.245 \\
\hline $\mathrm{TP}(\mathrm{g} / \mathrm{L})$ & $47.53 \pm 7.60$ & $49.17 \pm 8.28$ & $46.97 \pm 7.30$ & 0.056 \\
\hline $\operatorname{ALB}(g / L)$ & $24.69 \pm 5.42$ & $26.11 \pm 6.02$ & $24.20 \pm 5.11$ & $0.019 *$ \\
\hline GLO $(\mathrm{g} / \mathrm{L})$ & $22.92 \pm 4.61$ & $23.62 \pm 4.67$ & $22.68 \pm 4.58$ & 0.183 \\
\hline GLU (mmol/L) & $5.36 \pm 1.30$ & $5.59 \pm 1.99$ & $5.28 \pm 0.93$ & 0.248 \\
\hline $\mathrm{BUN}(\mathrm{mmol} / \mathrm{L})$ & $5.36 \pm 1.92$ & $5.44 \pm 1.93$ & $5.33 \pm 1.93$ & 0.702 \\
\hline CREA $(\mu \mathrm{mol} / \mathrm{L})$ & $64.78 \pm 15.78$ & $61.57 \pm 15.88$ & $65.90 \pm 15.64$ & $0.033^{*}$ \\
\hline Cys-C(mg/L) & $0.99 \pm 0.48$ & $1.06 \pm 0.81$ & $0.97 \pm 0.28$ & 0.427 \\
\hline $\mathrm{BMG}(\mathrm{mg} / \mathrm{L})$ & $2.32 \pm 0.80$ & $2.13 \pm 0.74$ & $2.39 \pm 0.81$ & $0.045^{*}$ \\
\hline RBP (mg/L) & $55.97 \pm 18.24$ & $54.36 \pm 16.44$ & $56.51 \pm 18.83$ & 0.451 \\
\hline $\mathrm{Ca}(\mathrm{mmol} / \mathrm{L})$ & $2.17 \pm 0.21$ & $2.17 \pm 0.21$ & $2.17 \pm 0.22$ & 0.972 \\
\hline $\mathrm{CHOL}(\mathrm{mmol} / \mathrm{L})$ & $8.84 \pm 2.68$ & $8.62 \pm 2.68$ & $8.92 \pm 2.69$ & 0.485 \\
\hline HDL-C (mmol/L) & $1.66 \pm 0.51$ & $1.71 \pm 0.60$ & $1.65 \pm 0.48$ & 0.454 \\
\hline LDL-C (mmol/L) & $5.45 \pm 2.25$ & $5.14 \pm 1.95$ & $5.56 \pm 2.34$ & 0.243 \\
\hline FSGS & $41 / 229(17.9 \%)$ & $9 / 59(15.25 \%)$ & $32 / 170(18.82 \%)$ & 0.538 \\
\hline $\mathrm{IgG} 4(\mathrm{mg} / \mathrm{L})$ & $352.49 \pm 317.78$ & $305.83 \pm 268.24$ & $368.56 \pm 332.43$ & 0.220 \\
\hline $\operatorname{IgG}(\mathrm{g} / \mathrm{L})$ & $5.74 \pm 2.51$ & $5.71 \pm 2.27$ & $5.74 \pm 2.59$ & 0.942 \\
\hline $\operatorname{IgM}(\mathrm{g} / \mathrm{L})$ & $1.13 \pm 0.51$ & $1.17 \pm 0.53$ & $1.11 \pm 0.50$ & 0.449 \\
\hline $\operatorname{IgA}(\mathrm{g} / \mathrm{L})$ & $2.34 \pm 0.91$ & $2.24 \pm 0.86$ & $2.37 \pm 0.86$ & 0.360 \\
\hline $\mathrm{C} 3(\mathrm{~g} / \mathrm{L})$ & $1.19 \pm 0.23$ & $1.19 \pm 0.18$ & $1.18 \pm 0.24$ & 0.937 \\
\hline $\mathrm{C} 4(\mathrm{~g} / \mathrm{L})$ & $0.30 \pm 0.08$ & $0.28 \pm 0.07$ & $0.30 \pm 0.18$ & 0.112 \\
\hline $\mathrm{Clq}(\mathrm{mg} / \mathrm{L})$ & $216.70 \pm 38.59$ & $213.19 \pm 34.61$ & $217.99 \pm 40.01$ & 0.463 \\
\hline
\end{tabular}

2

3

4 


\section{Table 2(on next page)}

Comparison of pathological characteristics between the two groups

Note: Compared with the PLa2R- group and the PLA2R+ group, $\mathrm{P} *<0.05, \mathrm{P} * *<0.01$. 
1 Table 2.

\begin{tabular}{llll}
\hline & $\begin{array}{l}\text { APLA2R-ab }(- \\
\text { (N=59) }\end{array}$ & $\begin{array}{l}\text { APLA2R-ab } \\
(+)(\mathbf{N}=\mathbf{1 7 0})\end{array}$ & P value \\
\hline Pathological stage (\%) & & & \\
I & $45 / 59(76.27 \%)$ & $112 / 170(65.88 \%)$ & 0.454 \\
II & $14 / 59(23.72 \%)$ & $55 / 170(32.35 \%)$ & 0.321 \\
III + IV & 0 & $2 / 170(1.18 \%)$ & 0.293 \\
Tissue IgG & $2.46 \pm 0.83$ & $2.37 \pm 0.89$ & 0.486 \\
Tissue IgM & $0.36 \pm 0.79$ & $0.38 \pm 0.69$ & 0.810 \\
Tissue IgA & $0.12 \pm 0.50$ & $0.23 \pm 0.61$ & 0.182 \\
Tissue C3 & $1.09 \pm 0.87$ & $1.19 \pm 0.94$ & 0.459 \\
Tissue Fib & $0.08 \pm 0.39$ & $0.15 \pm 0.52$ & 0.264 \\
Tissue C1q & $0.53 \pm 0.80$ & $0.50 \pm 0.71$ & 0.796 \\
Pathological characteristics & & & \\
Global sclerosis & $35 / 59(59.32 \%)$ & $88 / 170(51.76 \%)$ & 0.337 \\
Glomerular mesangial hyperplasia & $16 / 59(27.12 \%)$ & $64 / 170(37.65 \%)$ & 0.124 \\
Crescents & $2 / 59(3.38 \%)$ & 0 & 0.159 \\
Hyperplasia endothelialitis & $4 / 59(6.78 \%)$ & $7 / 170(4.12 \%)$ & 0.418 \\
Acute renal tubular lesions & $2 / 59(3.39 \%)$ & $20 / 170(11.76 \%)$ & $0.015^{*}$ \\
Chronic renal tubular lesions & $2 / 59(3.39 \%)$ & $21 / 170(13.53 \%)$ & $0.010^{* *}$ \\
Inflammatory cell infiltration & $3 / 59(5.08 \%)$ & $23 / 170(13.53 \%)$ & $0.031^{*}$ \\
Vascular disease & $21 / 59(35.59 \%)$ & $57 / 170(33.53 \%)$ & 0.796 \\
\hline
\end{tabular}

2

3 


\section{Table 3 (on next page)}

Comparison of remission rates after 1 year of follow-up and treatment in twogroups ofpatients

Note: CR, complete remission; PR, partial remission; CsA, cyclosporin; Pred, prednisone; TAC, tacrolimus; CTX, cyclophosphamide; MN, membranous nephropathy; Compared with the PLa2R-group and the PLA2R+ group, $\mathrm{P}^{*}<0.05, \mathrm{P}^{* *}<0.01$. 
Table3

\begin{tabular}{|c|c|c|c|}
\hline Total & $\begin{array}{ll}\text { Complete } & \text { remission } \\
\text { rate }(\mathrm{CR}) & \\
\end{array}$ & $\begin{array}{ll}\text { Partial remission } \\
\text { rate }(\mathrm{PR})\end{array}$ & CR+PR \\
\hline $\mathrm{MN}(\mathrm{N}=162)$ & $51 / 162(31.48 \%)$ & $78 / 1629(48.15 \%)$ & $129 / 162(79.63 \%)$ \\
\hline PLA2R- $(\mathrm{N}=43)$ & $22 / 43(51.16 \%)$ & $18 / 43(41.86 \%)$ & $40 / 43(93.02 \%)^{*}$ \\
\hline PLA2R+ $(\mathrm{N}=119)$ & $29 / 119(24.37 \%)$ & $60 / 119(50.42 \%)$ & $89 / 119(74.78 \%)$ \\
\hline \multicolumn{4}{|l|}{ PLA2R+ } \\
\hline low-titer(20-180 ) & $22 / 76(28.95 \%)$ & $40 / 76(52.63 \%)$ & $62 / 76(81.58 \%)^{*}$ \\
\hline high-titer(>180 ) & $7 / 43(16.28 \%)$ & 20/43(46.51\%) & $27 / 43(62.79 \%)$ \\
\hline \multicolumn{4}{|l|}{ Pred + Ctx } \\
\hline PLA2R- (N=15) & 9/15 (60\%) & 4/15 (26.67\%) & $13 / 15(86.67 \%)^{* *}$ \\
\hline $\mathrm{PLA} 2 \mathrm{R}+(\mathrm{N}=55)$ & $16 / 55(29.09 \%)$ & $24 / 55(45.45 \%)$ & $41 / 55(74.54 \%)$ \\
\hline \multicolumn{4}{|l|}{ Ped+Csa/ F506 } \\
\hline PLA2R- $(\mathrm{N}=25)$ & $11 / 25(44 \%)$ & $13 / 25(54.16 \%)$ & $24 / 25(96 \%)$ \\
\hline $\mathrm{PLA} 2 \mathrm{R}+(\mathrm{N}=61)$ & 12/61 (19.67\%) & $34 / 61(55.73)$ & $46 / 61(75.41 \%)$ \\
\hline \multicolumn{4}{|l|}{ Ped + Csa } \\
\hline PLA2R- $(\mathrm{N}=9)$ & 6/9 (66.67\%) & $3 / 9(33.33 \%)$ & 9/9 (100\%)* \\
\hline $\mathrm{PLA} 2 \mathrm{R}+(\mathrm{N}=31)$ & 6/31 (19.35\%) & $16 / 31(51.61 \%)$ & $22 / 31(70.97 \%)$ \\
\hline \multicolumn{4}{|l|}{ Ped+F506 } \\
\hline PLA2R- $(\mathrm{N}=16)$ & $5 / 16(31.25 \%)$ & $10 / 16(62.5 \%)$ & $15 / 16(93.75)$ \\
\hline PLA2R+ $(\mathrm{N}=30)$ & $6 / 30(20 \%)$ & $18 / 30(60 \%)$ & 24/30 (80\%) \\
\hline
\end{tabular}

2

3 
Table 4 (on next page)

Comparison of data between the two groups of patients after 1 year of follow-up treatment

Note: eGFR: estimated glomerular filtration rateCompared with the PLa2R-group and the $\mathrm{PLA} 2 \mathrm{R}+$ group, $\mathrm{P}^{*}<0.05, \mathrm{P} * *<0.01$. 
1 Table 4.

\begin{tabular}{|c|c|c|c|c|c|}
\hline Total & 24HUPRO (g/L) & $\operatorname{ALB}(g / L)$ & CREA $(\mu \mathrm{mol} / \mathrm{L})$ & BUN (mmol/L) & eGFR \\
\hline PLA2R-(N=43) & $0.78 \pm 1.10$ & $38.21 \pm 6.14$ & $64.18 \pm 14.37$ & $5.37 \pm 1.50$ & $107.77 \pm 24.43$ \\
\hline PLA2R+(N=119) & $2.22 \pm 2.10 * *$ & $35.09 \pm 6.97^{*}$ & $71.47 \pm 23.91$ & $6.68 \pm 2.72 *$ & $105.20 \pm 28.09$ \\
\hline \multicolumn{6}{|l|}{ Pred + CTx } \\
\hline PLA2R-(N=15) & $0.79 \pm 1.24$ & $37.37 \pm 6.80$ & $59.54 \pm 12.89$ & $4.83 \pm 1.23$ & $118.65 \pm 27.28$ \\
\hline $\mathrm{PLA} 2 \mathrm{R}+(\mathrm{N}=55)$ & $2.24 \pm 2.02$ & $34.28 \pm 6.46$ & $63.01 \pm 23.25$ & $5.75 \pm 1.68$ & $114.90 \pm 26.31$ \\
\hline PLA2R-(N=9) & $0.21 \pm 0.25$ & $40.01 \pm 4.49$ & $72.36 \pm 14.42$ & $6.25 \pm 2.20$ & $92.84 \pm 17.14$ \\
\hline $\mathrm{PLA} 2 \mathrm{R}+(\mathrm{N}=31)$ & $2.54 \pm 2.45^{* *}$ & $35.02 \pm 7.41$ & $80.85 \pm 23.66$ & $7.41 \pm 2.62$ & $93.74 \pm 23.15$ \\
\hline \multicolumn{6}{|l|}{ Ped+F506 } \\
\hline PLA2R-(N=16) & $1.18 \pm 0.61$ & $38.18 \pm 5.94$ & $67.77 \pm 15.70$ & $5.59 \pm 1.55$ & $115.02 \pm 28.47$ \\
\hline
\end{tabular}

2

3

4

5 


\section{Table 5 (on next page)}

Comparison of prognosis between the two groups 
1 Table 5.

Prognosis

Kidney death

Clinical death

Severe infection

Tumor

Complete remission

Partial remission

\section{PLA2R-(N=43)}

0

0

0

0

31

21

\section{PLA2R+(N=119)}

5

1

3

1

14

60

2

3 


\section{Table 6(on next page)}

Factors related to kidney death in patients with IMN (Cox single factor analysis)

Note: CRTL, chronic renal tubular lesions; ARTL, acute renal tubular lesions; $\beta 2-M G, \beta 2-$ microglobulin; 24HUPRO, 24-hour urine. 
吾 Table 6

\begin{tabular}{|c|c|c|c|c|c|c|c|c|}
\hline & \multirow[b]{2}{*}{ B } & \multirow[b]{2}{*}{ SE } & \multirow[b]{2}{*}{ Ward } & \multicolumn{2}{|c|}{ Degrees of } & \multirow[b]{2}{*}{$\operatorname{Exp}(B)$} & \multicolumn{2}{|c|}{$95 \% \operatorname{Exp}(\mathrm{B})^{\prime} \mathrm{Cl}$} \\
\hline & & & & freedom & Saliency & & Lower limit & Upper limit \\
\hline 24HUPRO & .236 & .096 & 6.100 & 1 & .014 & 1.266 & 1.050 & 1.527 \\
\hline$\beta 2-\mathrm{MG}$ & .651 & .249 & 6.822 & 1 & .009 & 1.917 & 1.176 & 3.123 \\
\hline CRTL & -1.880 & .817 & 5.298 & 1 & .021 & .153 & 1.322 & 32.516 \\
\hline ARTL & -2.169 & .818 & 7.038 & 1 & .008 & 1.114 & 1.762 & 43.468 \\
\hline
\end{tabular}




\section{Table 7 (on next page)}

Multivariate Cox regression analysis of renal death risk in patients with IMN

Note: CRTL, chronic renal tubular lesions; ARTL, acute renal tubular lesions; $\beta 2-M G, \beta 2-$ microglobulin; 24HUPRO, 24-hour urine. 
$1 \quad$ Table 7

\begin{tabular}{|c|c|c|c|c|c|c|c|c|}
\hline & \multirow[b]{2}{*}{ B } & \multirow[b]{2}{*}{ SE } & \multirow[b]{2}{*}{ Ward } & \multicolumn{2}{|c|}{ Degrees of } & \multirow[b]{2}{*}{$\operatorname{Exp}(B)$} & \multicolumn{2}{|c|}{$95 \% \operatorname{Exp}(\mathrm{B})^{\prime} \mathrm{Cl}$} \\
\hline & & & & freedom & Saliency & & Lower limit & Upper limit \\
\hline 24HUPRO & .291 & .138 & 4.483 & 1 & .034 & 1.338 & 1.022 & 1.752 \\
\hline$\beta 2-\mathrm{MG}$ & .971 & .532 & 3.334 & 1 & .068 & 2.641 & .931 & 7.491 \\
\hline CRTL & -1.937 & 1.607 & 1.453 & 1 & .228 & .144 & .006 & 3.360 \\
\hline ARTL & -.668 & 1.062 & .395 & 1 & .530 & 1.949 & .243 & 15.636 \\
\hline
\end{tabular}

4 
Figure 1

Detection of PLA2R antigen and IgG4 in renal tissue.

Note: The expression of PLA2R and IgG4 in glomeruli was observed by immunofluorescence microscopy in patients with negative anti-PLA2R antibodies. Simultaneous staining of PLA2R and IgG4 is positive were shown in A and B; Simultaneous staining of PLA2R and IgG4 is negative were shown in $C$ and $D ; E$ and $F$ show that PLA2R staining is negative and igG4 staining is positive. 


\section{PIA2R}
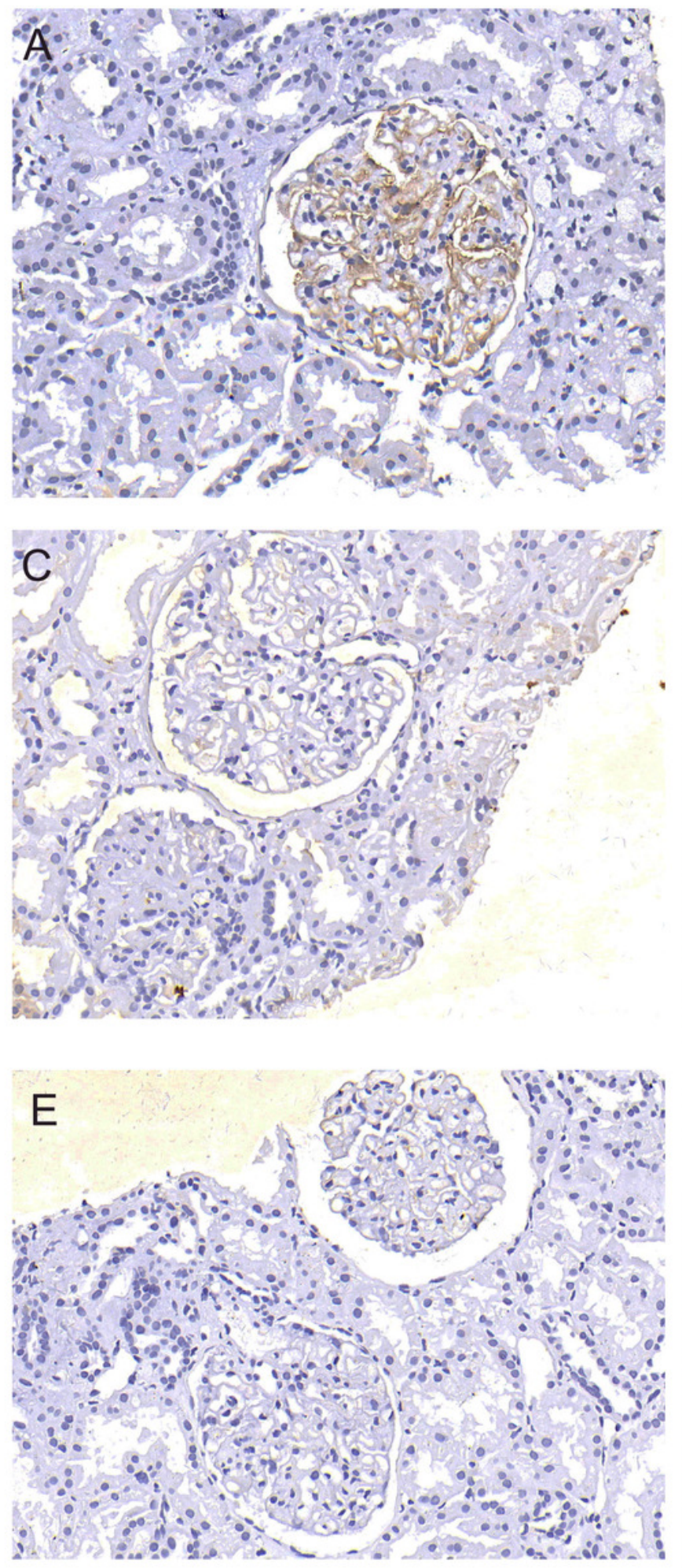

lgG4
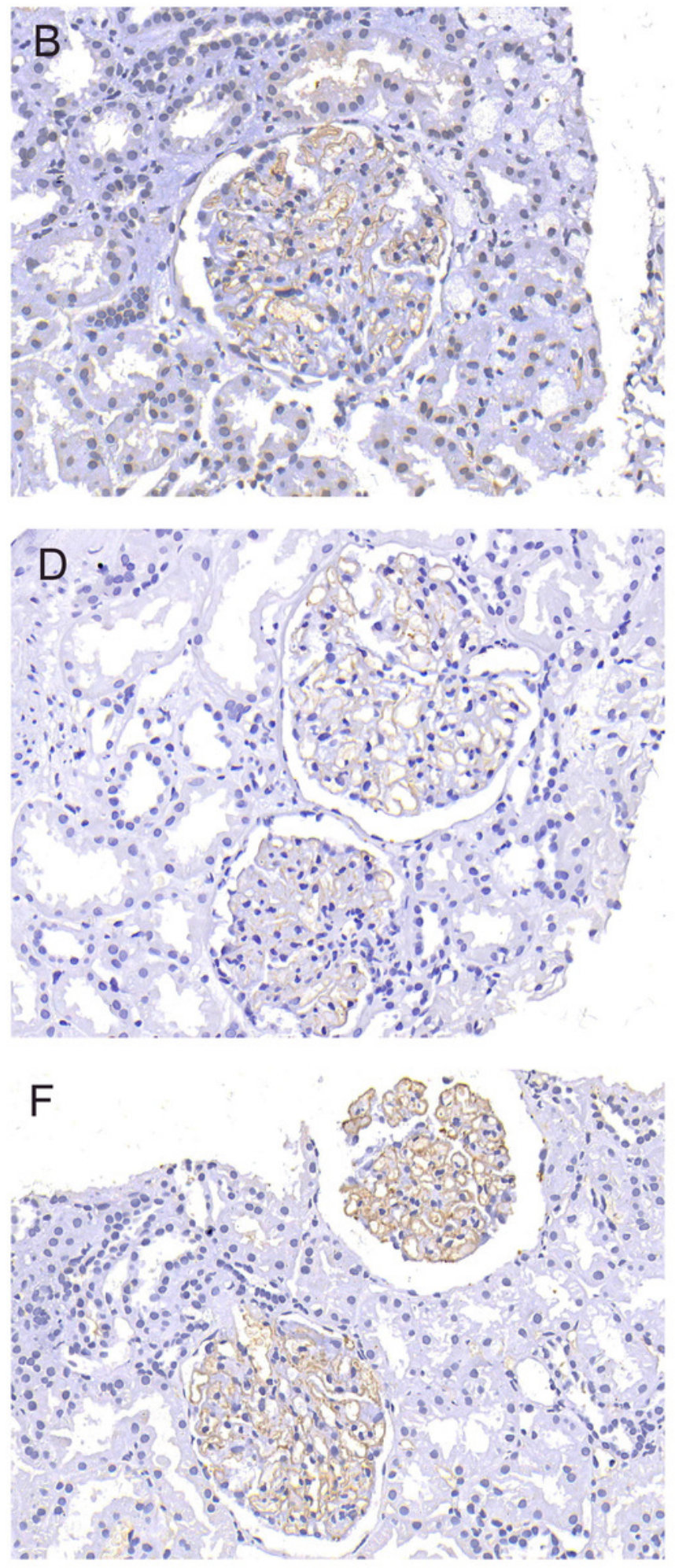
Figure 2

The log-rank test showed no significant difference in renal survival between the two groups.

The log-rank test showed no significant difference in renal survival between the two groups.

\section{Survival analysis curve}

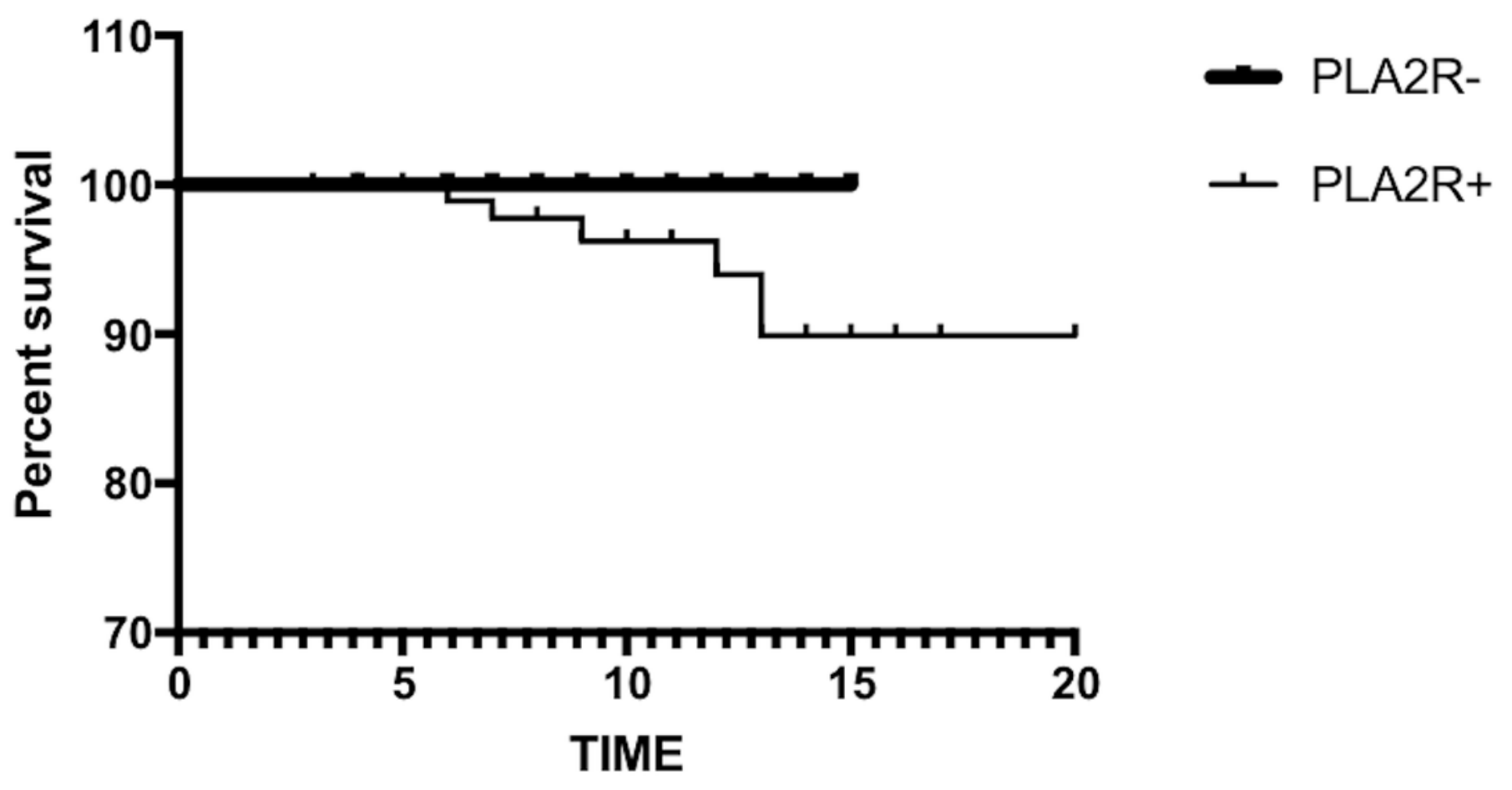

